\title{
Genomic and biochemical analysis of the diaminopimelate and lysine biosynthesis pathway in Verrucomicrobium spinosum: identification and partial characterization of $L, L$-diaminopimelate aminotransferase and UDP- $N$-acetylmuramoylalanyl-D-glutamyl-2,6-meso- diaminopimelate ligase
}

\author{
Victoria R. Nachar ${ }^{1}$, Francisco C. Savka ${ }^{1}$, Sean E. McGroty ${ }^{1}$, Katherine A. Donovan ${ }^{2}$, Rachel A. North ${ }^{2}$, \\ Renwick C. J. Dobson ${ }^{2,3}$, Larry J. Buckley ${ }^{1}$ and André O. Hudson ${ }^{1}$ * \\ ${ }^{1}$ The Thomas H. Gosnell School of Life Sciences, Rochester Institute of Technology, Rochester, NY, USA \\ 2 Biomolecular Interaction Centre, School of Biological Sciences, University of Canterbury, Christchurch, New Zealand \\ ${ }^{3}$ Department of Biochemistry and Molecular Biology, Bio21 Molecular Science and Biotechnology Institute, The University of Melbourne, Parkville, VIC, Australia
}

\section{Edited by:}

Naomi L. Ward, University of

Wyoming, USA

Reviewed by:

Uli Stingl, King Abdullah University of Science and Technology, Saudi Arabia

Evelyn Toh, Indiana University

Bloomington, USA

Yufeng Wang, University of Texas at

San Antonio, USA

*Correspondence:

André O. Hudson, The Thomas H.

Gosnell School of Life Sciences,

Rochester Institute of Technology, 85

Lomb Memorial Drive, Rochester NY

14623-5603, USA.

e-mail:aohsbi@rit.edu

The Gram-negative bacterium Verrucomicrobium spinosum has attracted interest in recent years following the sequencing and annotation of its genome. Comparative genomic analysis of $V$. spinosum using diaminopimelate/lysine metabolic genes from Chlamydia trachomatis suggests that $V$. spinosum employs the $L, L$-diaminopimelate aminotransferase (DapL) pathway for diaminopimelate/lysine biosynthesis. The open reading frame corresponding to the putative dapL ortholog was cloned and the recombinant enzyme was shown to possess $L, L$-diaminopimelate aminotransferase activity in vitro. In vivo analysis using functional complementation confirmed that the dapL ortholog was able to functionally complement an E. coli mutant that confers auxotrophy for diaminopimelate and Iysine. In addition to its role in lysine biosynthesis, the intermediate diaminopimelate has an integral role in peptidoglycan biosynthesis. To this end, the UDP-N-acetylmuramoylalanylD-glutamyl-2,6-meso-diaminopimelate ligase ortholog was also identified, cloned, and was shown to possess meso-diaminopimelate ligase activity in vivo. The L,L-diaminopimelate aminotransferase pathway has been experimentally confirmed in several bacteria, some of which are deemed pathogenic to animals. Since animals, and particularly humans, lack the genetic machinery for the synthesis of diaminopimelate/lysine de novo, the enzymes involved in this pathway are attractive targets for development of antibiotics. Whether dapL is an essential gene in any bacteria is currently not known. $V$. spinosum is an excellent candidate to investigate the essentiality of dapL, since the bacterium employs the DapL pathway for lysine and cell wall biosynthesis, is non-pathogenic to humans, facile to grow, and can be genetically manipulated.

Keywords: Iysine, diaminopimelate, L,L-diaminopimelate aminotransferase, peptidoglycan, functional complementation

\section{INTRODUCTION}

Verrucomicrobium spinosum is a rod-shaped heterotrophic Gramnegative bacterium that is found in fresh water and soil environments and is characterized as being non-motile and contains appendages known as prosthecae. V. spinosum is important to the biotechnology and medical sectors as it is closely related to Chlamydia (Wagner and Horn, 2006). A recent study confirmed that the organism contains all the genetic components to encode a Type III Secretion System and was able to kill Drosophila melanogaster and Caenorhabditis elegans, two model invertebrate hosts (Sait et al., 2011).

Genomic analysis of $V$. spinousm show that the organism contains all the genes necessary for the synthesis of diaminopimelate/lysine de novo and thus the bacterium is prototrophic for lysine. Lysine is synthesized using two general pathways that are evolutionarily divergent with respect to the intermediates used. One pathway utilizes the intermediate $\alpha$ amino adipic acid, which is derived from the metabolism of 2 -ketoglutarate a product of the citric acid cycle. The $\alpha$-amino adipic acid pathway is primarily used by fungi and is narrowly distributed to a few species belonging to the domain archaea (Nishida et al., 1999; Velasco et al., 2002). The other pathway employs the intermediate diaminopimelate, which is derived from oxaloacetate. The diaminopimelate pathway is found in most bacteria and photosynthetic organisms including cyanobacteria, algae, and plants. 
Four variants of the diaminopimelate/lysine pathway have been discovered so far (Figure 1): the two acyl pathways, which use either succinyl or acetyl intermediates; the mesodiaminopimelate dehydrogenase pathway; and the recently discovered $L, L$-diaminopimelate aminotransferasae (DapL) pathway (Hudson et al., 2006, 2008; McCoy et al., 2006). The biosynthesis of lysine from aspartate via the diaminopimelate/lysine pathway can be divided into three main events. The first event is the synthesis of tetrahydrodipicolinate from aspartate. This is a general feature of all four diaminopimelate pathway variants and is carried out by the enzymes, aspartate kinase, aspartate semialdehyde dehydrogenase, dihydrodipicolinate synthase, and dihydrodipicolinate reductase, respectively (Table 1 ). The second event constitutes the conversion of tetrahydrodipicolinate to the penultimate intermediate meso-diaminopimelate. The synthetic steps from tetrahydrodipicolinate to meso-diaminopimelate define the uniqueness of the diaminopimelate variant pathways. In the acyl pathways, four enzymes are required. These reactions are carried out by the enzymes 2,3,4,5-tetrahydropyridine-2,6dicarboxylate $N$-acyl-transferase, acyl-diaminopimelate aminotransferase, acyl-diaminopimelate deacylase, and diaminopimelate epimerase, respectively (Table 1, Figure 1). In the dehydrogenase pathway, tetrahydrodipicolinate is converted to mesodiaminopimelate by the enzyme meso-diaminopimelate dehydrogenase in one step circumventing the 2,3,4,5-tetrahydropyridine2,6-dicarboxylate-acyl-transferase, acyl-diaminopimelate aminotransferase, acyl-diaminopimelate deacylase, and diaminopimelate epimerase enzymatic reactions (Table 1, Figure 1). The $L, L$ diaminopimelate aminotransferase (DapL) pathway synthesizes
$L, L$-diaminopimelate from tetrahydrodipicolinate by a transamination reaction using glutamate as the amino donor and tetrahydrodipicolinate as the amino acceptor in a single reaction bypassing the 2,3,4,5-tetrahydropyridine-2,6-dicarboxylate -acyl-transferase, acyl-diaminopimelate aminotransferase, acyldiaminopimelate deacylase steps present in the succinylated or acetylated diaminopimelate/lysine pathways (Table 1, Figure 1). The third and ultimate event in the diaminopimelate variant pathways is defined by the enzyme meso-diaminopimelate decarboxylase which catalyzes the decarboxylation of meso-diaminopimelate to synthesize lysine. This decarboxylation step is shared by all four variants (Table 1, Figure 1).

Since animals do not contain the machinery to synthesize diaminopimelate or lysine, which are used by bacteria for peptidoglycan biosynthesis, the enzymes involved in the bacterial diaminopimelate/lysine biosynthesis pathways are of interest to the scientific community. meso-Diaminopimelate serves as one of the cross linking amino acids in the cell wall of Gramnegative bacteria, whereas lysine has the same role in Grampositive bacteria (Hutton et al., 2007). Inhibition of enzymes in the diaminopimelate/lysine biosynthesis pathways would have a detrimental effect to bacterial growth from two perspectives: since diaminopimelate is necessary for cell wall synthesis, cell lysis will occur as a result of osmotic pressure from the lack of peptidoglycan (Cox, 1996; Baizman et al., 2000); in addition, the inhibition of enzymes in the pathway will prevent protein synthesis, since lysine is one of the 20 common proteogenic amino acids. meso-Diaminopimelate is incorporated as one of the cross linking amino acids in the cell wall of Gram-negative bacteria

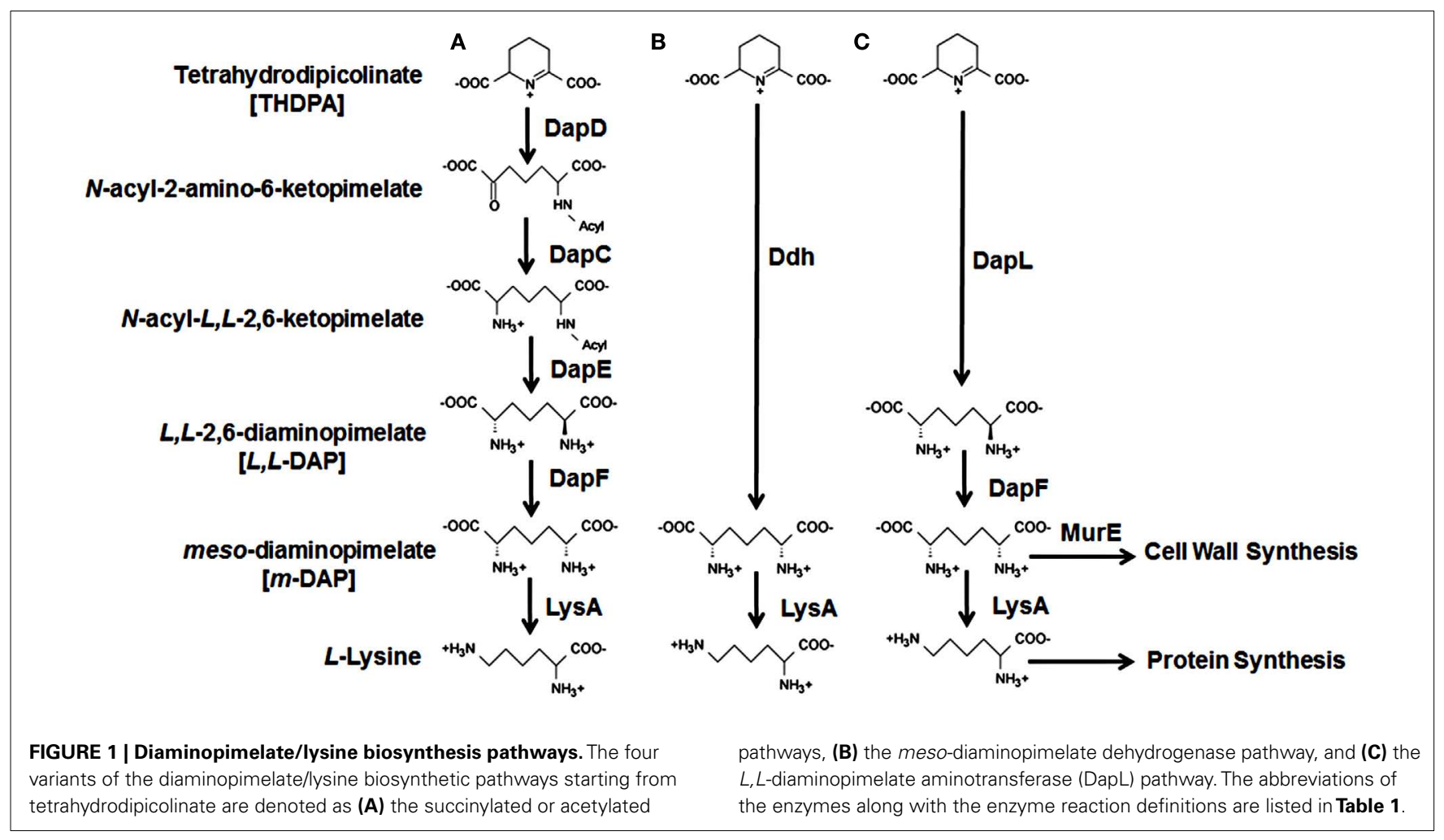


Table 1 | List of diaminopimelate/lysine biosynthesis genes from $V$. spinosum DSM $4136^{T}$.

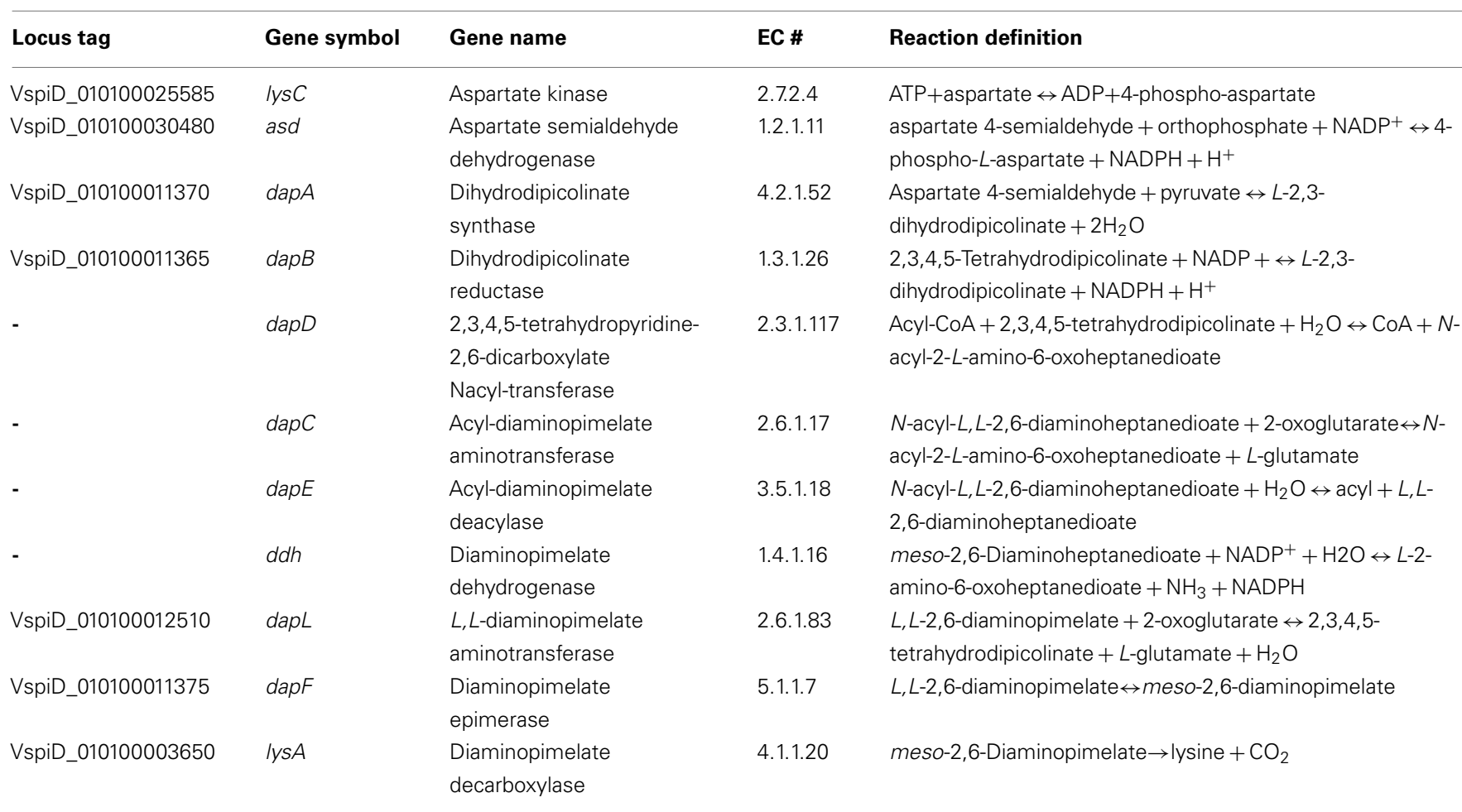

The list was generated using the genomic information deposited in the IMG database (http://img.jgi.doe.gov/cgi-bin/w/main.cgi).

by the enzyme UDP- $N$-acetylmuramoylalanyl-D-glutamyl-2,6meso-diaminopimelate ligase (MurE) (E.C. 6.3.2.15), which is encoded by the murE gene.

The DapL pathway has been identified in numerous bacteria and been experimentally confirmed in a limited number of bacteria, some of which are deemed pathogenic (McCoy et al., 2006; Hudson et al., 2008; Liu et al., 2010). The three-dimensional crystal structure of DapL from Arabidopsis thaliana has been solved (Watanabe et al., 2007, 2008) from Chlamydia trachomatis (Watanabe et al., 2011) and from the algae Chlamydomonas reinhardtii (Dobson et al., 2011; Hudson et al., 2011a). Inhibitors for the Arabidopsis DapL ortholog have been reported using in vitro studies (Fan et al., 2010). It should be noted that although inhibitors the Arabidopsis DapL have been found, it is not known if these compounds inhibit other aminotransferases.

The identification of dapL and murE genes, in addition to the easy culturability and genetic manipulation of $V$. spinosum, makes the bacterium an excellent model organism for experiments addressing the essentiality of dapL and murE with respect to lysine and peptidoglycan biosynthesis (Domman et al., 2011). Most bacterial species that contain the DapL pathway are difficult to work with, since they are either slow growing, difficult to culture, anaerobic, and pathogenic to humans.

Here we present the first genomic and biochemical analysis of the diaminopimelate/lysine biosynthesis pathway from V. spinosum. We identify the genes necessary for diaminopimelate/lysine biosynthesis in the genome of $V$. spinosum and demonstrate that the bacterium uses the recently discovered DapL pathway for diaminopimelate/lysine biosynthesis. In addition, we identify and characterize MurE from V. spinosum, suggesting that the bacterium incorporates meso-diaminopimelate into its peptidoglycan as a cross linking amino acid.

\section{RESULTS}

IDENTIFICATION OF PUTATIVE dapL AND murE GENES IN V. SPINOSUM

The dapL ortholog from $V$. spinosum was identified using the DapL protein from C. trachomatis Ct390 (NP_219900) as the query with the BlastP algorithm from the Integrated Microbial Genomes (IMG) database ${ }^{1}$. This search identified a putative $L, L$ diaminopimelate aminotransferase annotated by the locus tag VspiD_010100012510 (ZP_02927470) that is 38\% amino acid identity to the $C$. trachomatis DapL. The murE ortholog was identified using the C. trachomatis MurE (NP_219774) as a query. This search resulted in the identification of the murE ortholog from $V$. spinosum annotated by the locus tag VspiD_010100019130, which is $37 \%$ identical to the C. trachomatis MurE.

\section{CRUDE SOLUBLE PROTEIN EXTRACT FROM V. SPINOSUM CONTAINS DapL ACTIVITY}

If $V$. spinosum utilizes the DapL pathway for diaminopimelate/lysine biosynthesis, as suspected by the genomic analysis, a crude soluble protein extract should possess DapL activity. The ortho-aminobenzaldehyde assay was employed using a crude soluble protein extract from $V$. spinosum. Using $L, L$-diaminopimelate

\footnotetext{
${ }^{1}$ http://www.jgi.doe.gov/
} 
as the amino donor and 2-ketoglutarate as the amino acceptor, the assay measures the production of dihydroquinazolium which is the product of the interaction between tetrahydrodipicolinate and ortho-aminobenzaldehyde. DapL activity was detected from the crude soluble extract showing that the rate of the reaction is proportional to the amount of $V$. spinosum protein extract added to the assay (Figure 2). Enzymatic activity was not observed when the amino donor, or amino acceptor, was omitted from the assay. In addition, no activity was observed when the protein extract was heated in boiling water for $5 \mathrm{~min}$.

\section{DapL IS THE ONLY ROUTE FOR DIAMINOPIMELATE/LYSINE BIOSYNTHESIS IN V. SPINOSUM}

The genome of $V$. spinosum was searched to catalog the genes necessary for the de novo anabolism of lysine from aspartate. Since DapL activity was detected from a soluble extract, we wanted to know whether the DapL pathway was the sole route toward lysine biosynthesis. There are examples in the literature of multiple pathways for lysine biosynthesis in bacteria; for example, the genomes of Bacteroides fragilis and Clostridium thermocellum were found to contain both the DapL and mesodiaminopimelate dehydrogenase pathways (Hudson et al., 2011b). Also, Corynebacterium glutamicum employs the acyl pathway in conjunction with the Ddh pathway (Schrumpf et al., 1991). A search of the $V$. spinosum genome suggests that the DapL pathway is the only route for diaminopimelate/lysine biosynthesis, since orthologs of the acyl and dehydrogenase genes could not be identified in the genome (Table 1; Figure 1). Even though the search did not identify a diaminopimelate dehydrogenase ortholog, we tested for dehydrogenase activity using the same protein extract that was used to detect DapL activity. This test was done using $1.0 \mathrm{mg}$ of total protein in two different buffer systems, finding no activity. The recombinant diaminopimelate dehydrogenase from Clostridium thermocellum was used as a positive control (Hudson et al., 2011b). This result was

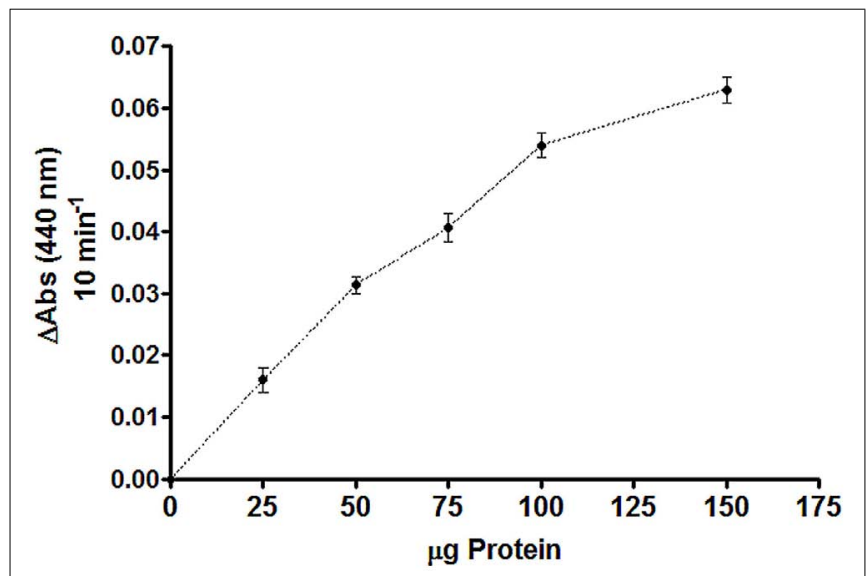

FIGURE 2 | DapL activity from crude protein extract from $\boldsymbol{V}$. spinosum. The graph shows the relationship between the protein amount and reaction rate. The DapL ortho-aminobenzaldehyde assay is described in the Section "Materials and Methods." The assay was done in triplicates for each protein concentration. consistent with the genomic analysis regarding the absence of a diaminopimelate dehydrogenase ortholog in the genome of $V$. spinosum.

\section{THE GENE ANNOTATED BY THE LOCUS TAG VspiD_010100012510 (VsdapL) ENCODES AN AUTHENTIC L,L-DIAMINOPIMELATE AMINOTRANSFERASE}

The putative VsdapL gene was cloned and the recombinant enzyme was purified to homogeneity using affinity chromatography (Figure 3). The ortho-aminobenzaldehyde assay was used to determine whether the putative $V s$ DapL had $L, L$-diaminopimelate aminotransferase activity. The results from this analysis show that the open reading frame annotated by the locus tag encodes an authentic $L, L$-diaminopimelate aminotransferase enzyme. With $L, L$-diaminopimelate as the amino donor and 2-ketoglutarate as the amino acceptor, the specific activity of the $V s$ DapL is $4.1 \pm 0.25$ $\Delta A_{440} \mathrm{~min}^{-1} \mathrm{mg}^{-1}$. Unlike the plant and algae enzyme, $V s \mathrm{DapL}$ possesses transamination activity with several diamine donors that are structurally similar to $L, L$-diaminopimelate, including the racemic isomer meso-diaminopimelate (Table 2 ), albeit at relatively low rates. The enzyme is also able to use several oxoacids as amino acceptors in addition to 2-ketoglutarate (Table 2); again the relative rates are low.

\section{VsdapL IS ABLE TO FUNCTIONALLY COMPLEMENT THE E. COLI $\triangle$ dapD/dapE (AOH1) MUTANT}

The E. coli strain AOH1 harbors a deletion of the dapD gene and a mutation in $d a p E$ that renders this enzyme nonfunctional (Hudson et al., 2006). As such, the mutant is unable to synthesize meso-diaminopimelate for lysine and cell wall biosynthesis. As a result, the cells lyse from osmotic pressure due to the lack of meso-diaminopimelate as a cross linking amino acid in the cell wall and is deemed auxotrophic for diaminopimelate and lysine. For complementation analysis, E. coli AOH1 was transformed with the empty vector (pBAD33), or with the DapL expression vectors (pBAD33 + dapL) from the plant Arabidopsis thaliana (AtdapL; Hudson et al., 2006), the alga C. reinhardtii (CrdapL; Dobson et al., 2011) or the bacterium V. spinosum (VsdapL). While E. coli AOH1 is able to grow on media supplemented with $L, L$-diaminopimelate, only the auxotrophic mutant expressing authentic orthologous

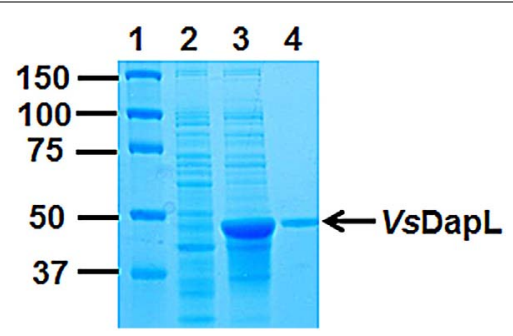

FIGURE 3 | Recombinant expression and purification of $V s$ DapL using affinity chromatography. Lane (1) Protein Marker (kDa), Lane (2) $10 \mu \mathrm{g}$ of soluble protein from uninduced cells, Lane (3) $10 \mu \mathrm{g}$ of soluble proteins from induced cells, Lane (4) $1 \mu \mathrm{g}$ of purified VsDapL. The proteins were resolved on a $10 \%(\mathrm{w} / \mathrm{v})$ acrylamide gel and were stained with Coomassie blue for visualization. 
DapLs are able to grow on $L, L$-diaminopimelate-free media (Figure 4). This analysis demonstrates that the recombinant enzymes are able to convert tetrahydrodipicolinate to $L, L$ diaminopimelate, bypassing three acyl enzymatic reactions present in the E. coli acyl pathway, to facilitate the synthesis of mesodiaminopimelate for lysine and cell wall biosynthesis (Figures 1 and 5).

\section{VsDapL BELONGS TO THE DAPL1 FORM OF L,L-DIAMINOPIMELATE AMINOTRANSFERASES}

Diaminopimelate aminotransferases are approximately 400 amino acids in length and are members of the pyridoxal-5' phosphate dependent family of class I/II transaminases. A recent study revealed that there are two diverged forms of DapL enzymes; DapL1 and DapL2 (Hudson et al., 2008). The two forms of DapLs share approximately $30 \%$ homology on the amino acid level. The DapL ortholog from C. trachomatis is evolutionarily related to DapL1 enzymes (Hudson et al., 2008). Since the homology of the V. spinosum DapL is only 38\% to the C. trachomatis ortholog, the form of the V. spinosum DapL was not apparent. Using phylogenetic analysis, the DapL ortholog from $V$. spinosum clusters with enzymes some of which have been experimentally confirmed as DapL1 orthologs and from pathogenic organisms (Hudson et al., 2008, 2011b). The analysis shows two distinct clades, DapL1, DapL2. The aspartate aminotransferase from Yersinia pseudotuberculosis, which is also classified as a class I/II transaminase is used as an outgroup (Figure 6). Four analyses were performed (ML, MP, ME, NJ) and all show moderate to strong support the DapL2 clade (57-100\%) and for the DapL1 clade (56-99\%). The more variable maximum likelihood bootstrap support is alignment dependent with alternative alignments techniques (Clustal vs. MUSCLE) exhibiting higher ML bootstrap values for the DapL1 and DapL2 clades respectively. Muscle-aligned sequences generally exhibit higher bootstrap support for both DapL1 and DapL2 clades than Clustal.

\section{VsmurE IS ABLE TO FUNCTIONALLY COMPLEMENT THE E. COLI murE MUTANT}

meso-Diaminopimelate serves not only as the penultimate precursor for lysine biosynthesis, but it is also incorporated as one of the cross linking amino acids in the cell wall of Gramnegative bacteria. This is facilitated by the enzyme UDP- $N$ acetylmuramoylalanyl- $D$-glutamyl-2,6-meso-diaminopimelate ligase (MurE; Figure 5). Since V. spinosum is classified as Gramnegative bacterium, it should possess a MurE ortholog (Schlesner et al., 2006). The VsmurE ortholog was identified and the open reading frame was cloned into an expression vector to test via functional complementation whether the gene encodes an authentic MurE enzyme. The E. coli mutant TKL-11 harbors a mutation in the murE gene which results in a temperature sensitive growth phenotype where the mutant is able to grow at the permissive temperature of $30^{\circ} \mathrm{C}$, but not at $42^{\circ} \mathrm{C}$ (Lugtenberg and van Schijndel-van Dam, 1972). The mutant was transformed with an empty vector (pBAD33) and a vector expressing the putative MurE (pBAD33 + VsmurE). Using replica-plating, the results from this analysis demonstrate that at the permissive temperature of $30^{\circ} \mathrm{C}$, the mutant harboring both the vector control and the vector expressing the recombinant enzyme are able to grow. However, when exposed to the non-permissive temperature of $42^{\circ} \mathrm{C}$, only the mutant expressing the MurE ortholog is able to grow (Figure 7A). This result was corroborated by assessing bacterial growth over a period of $10 \mathrm{~h}$. At $30^{\circ} \mathrm{C}$ the mutant harboring the vector only and the murE expression vector grew as expected. However, when the cultures were switched to the non-permissive temperature of $42^{\circ} \mathrm{C}$ after $5 \mathrm{~h}$ from $30^{\circ} \mathrm{C}$, only the mutant harboring the murE expression vector continued to grow. The optical density of the vector only culture declined which can be attributed to rapid lysis of the cell due to the lack of proper peptidoglycan synthesis (Figure 7B). The assessment of crude soluble protein extracts from the complementation experiment using SDS-PAGE analysis confirmed the expression of the recombinant MurE $(59 \mathrm{kDa})$ in TKL-11 cells harboring the expression vector (pBAD33 + VsmurE) grown at
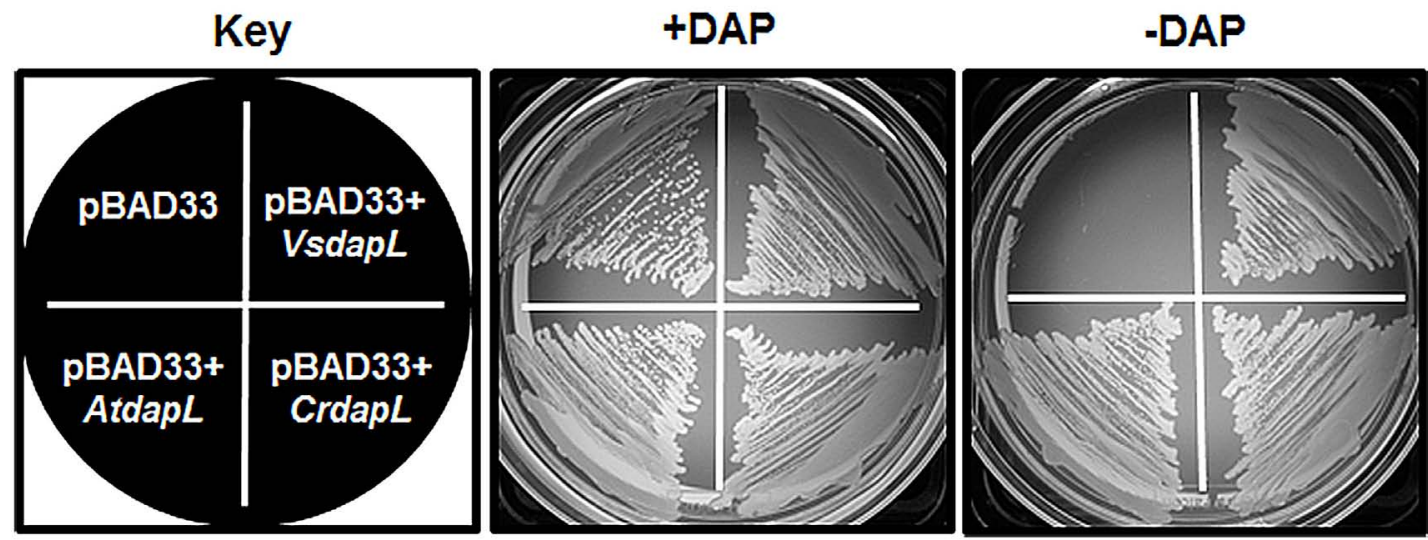

FIGURE 4 | Functional complementation of $\boldsymbol{E}$. coli $\mathbf{\Delta} \boldsymbol{d a p D / d a p E}(\mathbf{A O H} 1)$ mutant. The AOH1 mutant harboring the plasmids pBAD33, pBAD33 + AtdapL, pBAD33 + CrdapL, and pBAD33 + VsdapL was replica-plated on LB medium supplemented with $0.2 \%$ (w/v) arabinose with or without $50 \mu \mathrm{g} / \mathrm{mL}$ $L, L$-diaminopimelate. For presentation purposes only one colony from the vector only and recombinant plasmids are represented in the diagram. 


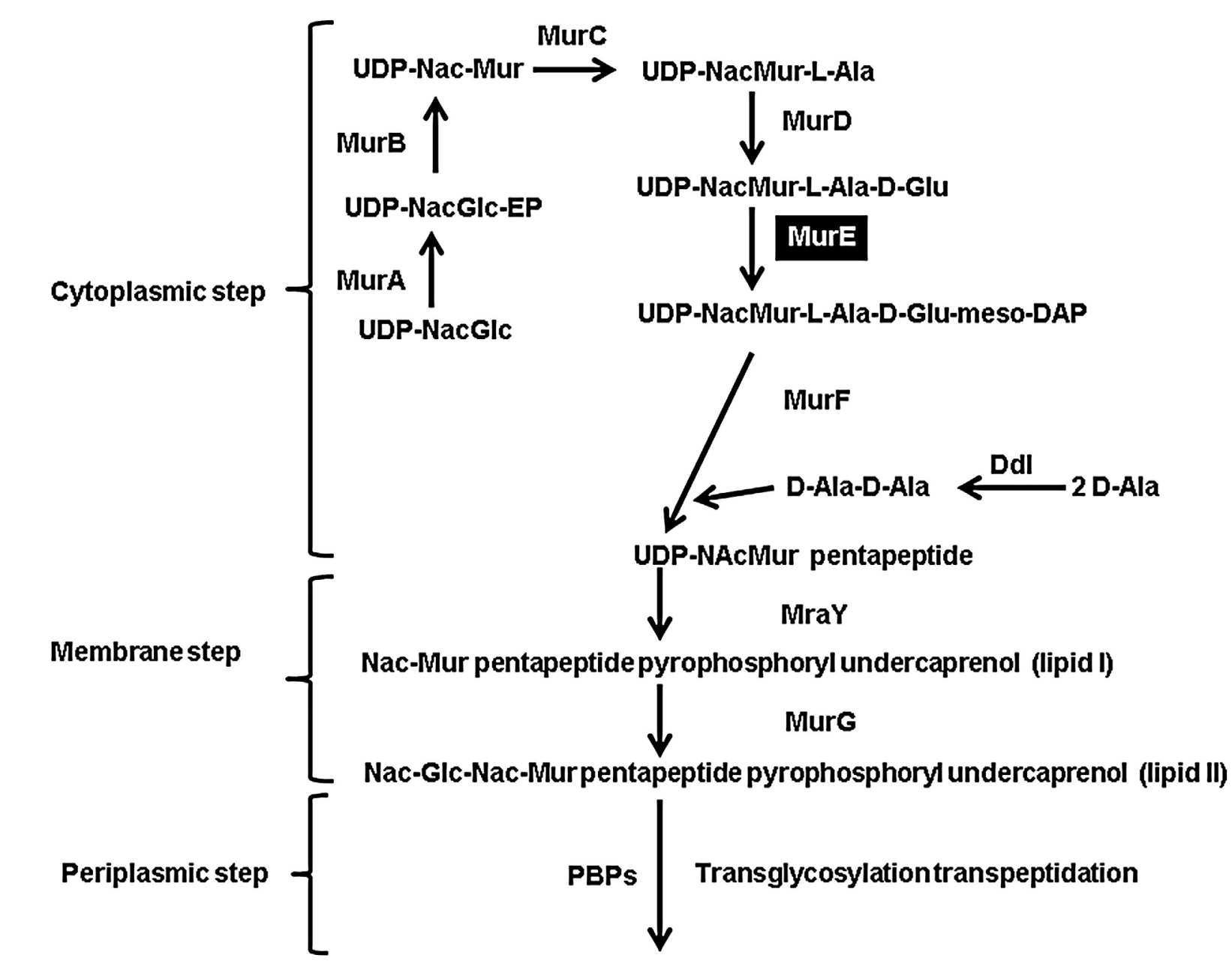

FIGURE 5 | Schematic representation of the bacterial peptidoglycan synthesis using $\boldsymbol{m}$-DAP as one of the cross linking amino acids. The cytosolic, membrane, and periplasmic steps are shown. The abbreviations of the enzymes are as follows: MurA (UDP-N-acetylglucosamine enolpyruvyl transferase), MurB (UDP- $N$-acetylenolpyruvoylglucosaminereductase), MurC (UDP- $N$-acetylmuramate- $L$-alanine ligase), MurD (UDP- $N$-acetyl-
muramoylalanine-D-glutamate ligase), MurE (UDP-N-acetylmuramoylL-alanyl-D-glutamate 2,6-meso-diaminopimelate ligase), MurF (UDP-N-acetylmuramoyl-tripeptide-D-alanyl-d-alanine ligase), Ddl (D-alanine-D-alanine ligase B), MraY (phospho- $N$-acetylmuramoylpentapeptide-transferase), MurG (pyrophosphoryl-undecaprenol$\mathrm{N}$-acetylglucosamine transferase), and PBPs (penicillin binding proteins). $42^{\circ} \mathrm{C}$ that is not present in the extract from TKL-11 harboring the vector only control (pBAD33) grown at $30^{\circ} \mathrm{C}$ (Figure 7C).

\section{THE AMINO ACIDS THAT CONSTITUTE THE ACTIVE SITE ARE CONSERVED IN VsDapL}

To examine more closely the $V s$ DapL variant at the amino acid level, we aligned its amino acid sequence to that of three DapL orthologs already functionally and structurally characterized from Arabidopsis thaliana (Watanabe et al., 2007), C. reinhardtii (Dobson et al., 2011), and C. trachomatis (Watanabe et al., 2011; Figure 8). The $V s \mathrm{DapL}$ sequence has more similarity to the CrDapL and AtDapL sequences, but like the CtDapL sequence, has a truncated $\mathrm{N}$-terminal region. The longer $\mathrm{N}$-terminal regions for the eukaryotic sequences is attributed to subcellular localization sequences, used by $A$. thaliana and $C$. reinhardtii to target the enzyme to the chloroplast, where lysine biosynthesis in known to occur (Mills and Wilson, 1978). As can be seen in Figure 8 (residues in red bold), the putative active site residues are well conserved between the four DapL enzymes. In addition, the loop regions that form the active site cleft are generally well conserved. In particular, loops $\mathrm{A}$ and $\mathrm{C}$ are well conserved, although loop B shows more variability. From previous structural studies (Watanabe et al., 2007, 2011; Dobson et al., 2011), it is known that loop B is disordered and its role in catalysis is less clear. However, noting the increased substrate promiscuity displayed by $V s \mathrm{DapL}$, it is possible that loop B may be involved in the substrate binding step.

A homology model of the $V s$ DapL was generated using the Swiss-Model Protein Modeling Server (Figure 9). The tertiary structure of the DapL monomer has been annotated as having a large and a small domain, which also includes the arm region (Figure 9A). Most aminotransferases are approximately $100 \mathrm{kDa}$ in mass, representing a homodimeric quaternary structure. Assuming that the functional unit of the VsDapL enzyme is 


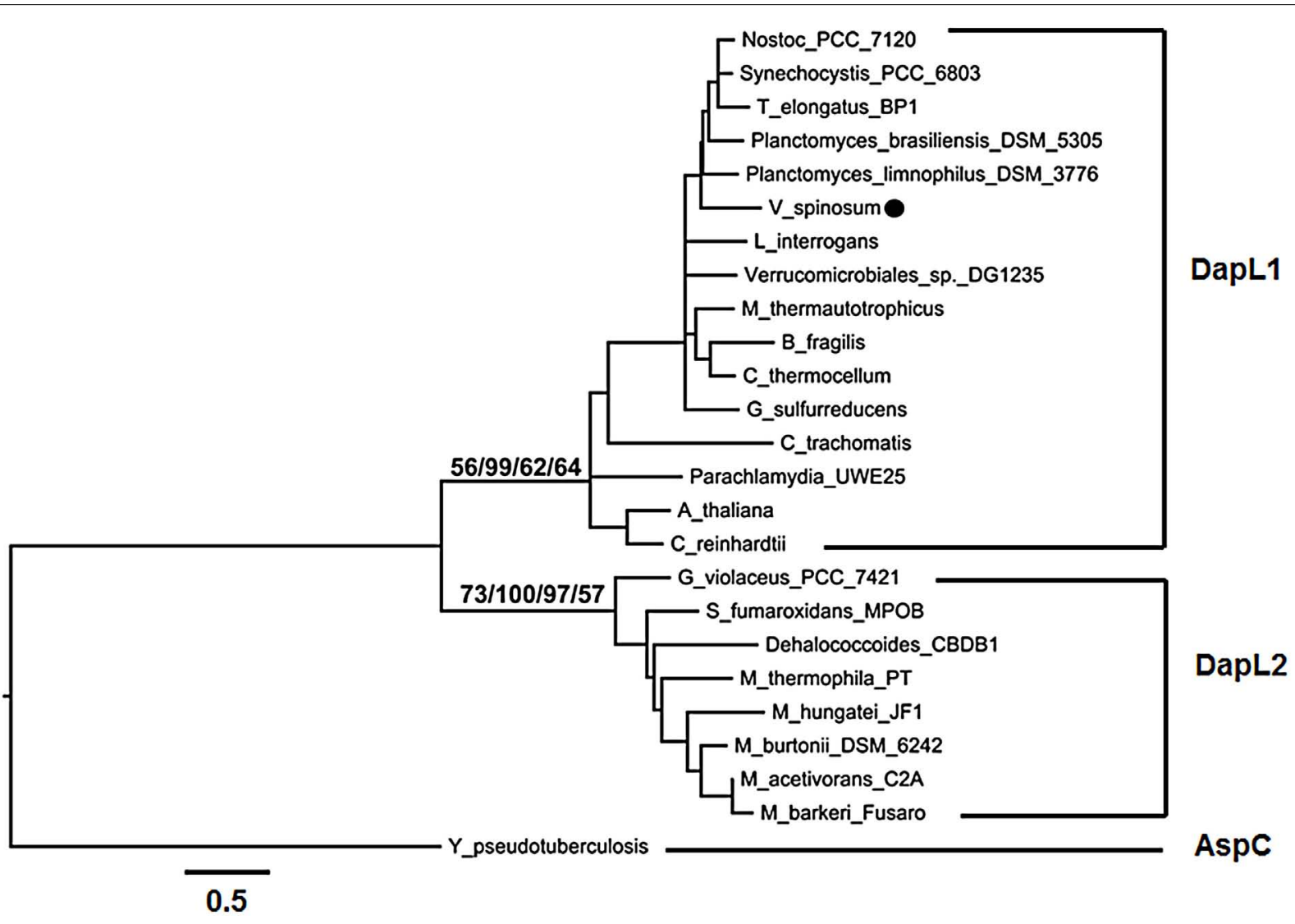

FIGURE 6 | Phylogenetic relationship of DapL amino acid sequences.

The VsDapL ortholog is annotated with a black circle. Minimum evolution (ME) topology shown with maximum likelihood (ML), maximum parsimony (MP), minimum evolution (ME), and neighbor-joining (NJ) bootstrap values for both the DapL1 and DapL2 clades (from left to right).
Branch lengths are proportional to divergence; the scale below the tree indicates percent divergence of the branch lengths. The tree was rooted with a single outgroup aspartate aminotransferase (AspC) from

Y. pseudotuberculosis. Figure drawing produced using FigTree (ver. 1.6.1; Rambaut, 2009). a dimer, which is required for proper orientation of the conserved active site residues within the active site, $V s$ DapL probably has two active sites (Figure 9B). Closer examination of one active site (Figure 9C) highlights those residues that are conserved from the multiple sequence alignment (Figure 8) and the key loop regions that line the active site. Importantly, the model predicts that loop $\mathrm{B}$ is positioned at the entrance of the active site cleft, ideally placed to interact with substrates entering the active site.

\section{DISCUSSION}

The discovery of a new biosynthetic route to diaminopimelate and lysine in plants, algae, and bacteria provides a fresh target for the discovery of novel herbicides, algaecides, and antibiotics. The amino acids that constitute the active site of DapL are conserved in the V. spinosum ortholog based on protein alignment and modeling. Therefore, inhibitors of CtDapL and AtDapL are likely to inhibit VsDapL. To this end, V. spinosum is a suitable bacterial system for the development of in vivo assays for the discovery of compounds that are able to inhibit DapL to facilitate antibiotic development.

Since $V$. spinosum can be genetically altered using exogenous DNA, $V$. spinosum is an excellent candidate for determining the essentiality of dapL via mutagenic experiments using transposon and or homologous recombination. Moreover, the organism is aerobic, relatively easy to culture and non-pathogenic to humans, unlike other bacteria that contain the DapL pathway, such as Leptospira interrogans, Bacteriodes fragilis, and C. trachomatis (McCoy et al., 2006; Hudson et al., 2008).

Recently, our laboratories demonstrated that the orthologous dapL from the model plant system Arabidopsis thaliana is essential for growth and development (Dobson et al., 2011). However, it is not known if the same is true for bacteria that exclusively utilize the DapL pathway for diaminopimelate/lysine synthesis. It is not known whether another aminotransferase capable of interconverting tetrahydrodipicolinate and $L, L$-diaminopimelate exists in bacteria that solely contain the DapL pathway. Thus, a suitable dapL bacterial mutant that is auxotrophic for diaminopimelate/lysine would be an important step forward especially since there are examples in the literature regarding substrate promiscuity of aminotransferases involved in amino acid metabolism. For instance, the aspartate aminotransferase, tyrosine aminotransferase, and the branched-chain amino acid aminotransferase have been shown to possess overlapping activities in E. coli $(\mathrm{Gu}$ et al., 1998). In addition, a recent study demonstrated that three 


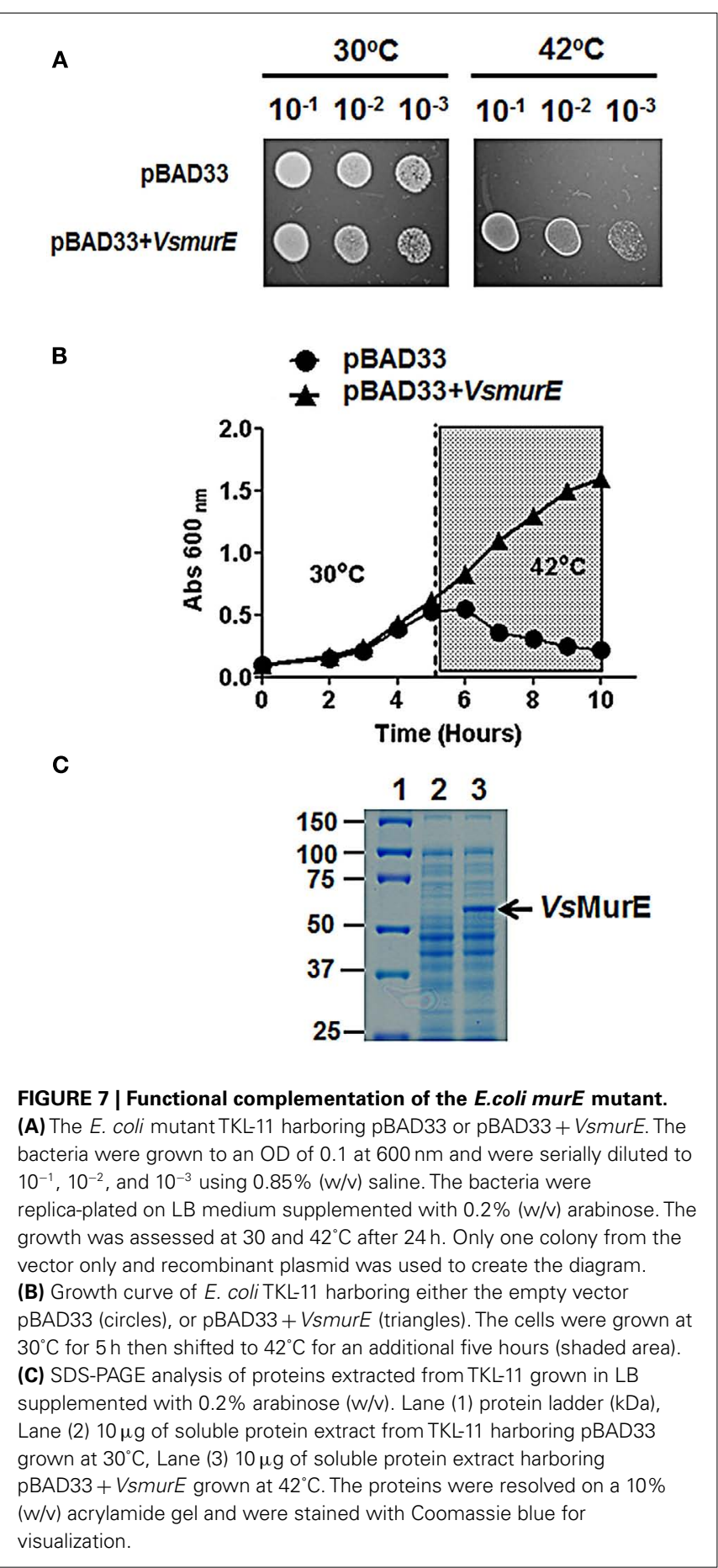

aminotransferases are involved in alanine biosynthesis in E. coli (Yoneyama et al., 2011).

Aminotransferases are common in the genome of $V$. spinosum given their integral role in multiple anabolic and catabolic pathways. If a compound inhibits a bacterial DapL in vitro, as in the case of the Arabidopsis enzyme, the question of whether this compound is specific for DapL in vivo will have to be investigated. One way to answer this question would be to use in vivo studies by exposing the putative inhibitory compound to a dapL mutant supplemented with diaminopimelate in the medium to observe the growth phenotype. If the inhibitory compound is specific for DapL, one would expect that it will not have any effect on the mutant. However, the same compound should have an effect on the wild-type parental strain used to create the dapL mutant. The inhibition of DapL will prevent $m$-DAP production which would lead to cell death caused by the lack of proper protein and cell wall biosynthesis.

Here we present the elucidation of the biosynthetic pathway of diaminopimelate/lysine from V. spinosum using genomic, biochemical, and structural approaches. The enzymes involved in the bacterial diaminopimelate/lysine biosynthetic pathways are presumed targets for antibiotic development. The identification and characterization of these enzymes provides valuable information pertaining to experiments aiming to elucidate the essentiality of genes involved in the bacterial diaminopimelate/lysine biosynthesis pathways. The DapL pathway is the sole route toward diaminopimelate/lysine in V. spinosum. In addition, the bacterium can be genetically modified, the organism is relatively easy to culture and non-pathogenic to animals. These criteria make V. spinosum an excellent bacterial model for elucidating the essentiality of genes involved in diaminopimelate/lysine metabolism.

\section{MATERIALS AND METHODS V. SPINOSUM GROWTH CONDITIONS}

The plasmids and strains used in this study are listed in Table 3. The V. spinosum DSM $4136^{\mathrm{T}}$ organism was cultured in R2A medium at $26^{\circ} \mathrm{C}$.

\section{MULTIPLE-PROTEIN SEOUENCE ALIGNMENT, PHYLOGENETIC TREE CONSTRUCTION, AND HOMOLOGY MODEL OF VsDapL}

A DapL protein sequence alignment was generated using the ClustalW server ${ }^{2}$. Phylogenetic trees were constructed from MUSCLE aligned amino acid sequences using maximum likelihood $(\mathrm{ML})$, maximum parsimony (MP) minimum evolution (ME), and neighbor-joining (NJ) techniques in MEGA 5.05 (Tamura et al., 2011). ML searches were performed using the likelihoodbased WAG (Whelan and Goldman, 2001) model of amino acid character evolution, gamma distributed rates across sites, and the proportion of invariant sites estimated from the data set $(\mathrm{WAG}+\mathrm{G}+\mathrm{I})$. ME and NJ tree searches were performed using the parsimony-based JTT model (Jones et al., 1992) and gamma distributed rates $(\mathrm{JTT}+\mathrm{G})$. Optimal models were determined by MEGA 5.05 that iteratively tests the partition against hierarchically nested models of evolution to obtain the best fit. Node strength was assessed using the bootstrap technique and 100 pseudo-replicate data sets for each analysis type (ML, MP, ME, and $\mathrm{NJ})$. The accessions numbers for the following DapL proteins are: Arabidopsis thaliana (AEE86265), V. spinosum (ZP_02927470), C. reinhardtii (XP_001693061), C. trachomatis (NP_219900), Parachlamydia UWE25 (YP_007684), Synechocystis sp. PCC 6803 (BAA10583), Thermosynechococcus elongatus BP1 NP_682892), Bacteroides fragilis NCTC 9343 (YP_212286), Clostridium thermocellum 27405 (YP_001039489), Methanothermobacter

\footnotetext{
${ }^{2}$ http://www.ebi.ac.uk/Tools/msa/clustalw2/
} 
V.spinosum-DapL

C. trachomatis-DapL

A. thaliana-DapL

C. reinhardti-DapL

V.spinosum-DapL

C. trachomatis-DapL

A. thaliana-DapL

C.reinhardti-DapL

V.spinosum-DapL

C. trachomatis-DapL

A. thaliana-DapL

C.reinhardti-DapL

V.spinosum-DapL

C. trachomatis-DapL

A. thaliana-DapL

C.reinhardti-DapL

V. spinosum-DapL

C. trachomatis-DapL

A. thaliana-DapL

C.reinhardti-DapL

V.spinosum-DapL

C. trachomatis-DapL

A. thaliana-DapL

C.reinhardti-DapL

V. spinosum-DapL

C. trachomatis-DapL

A. thaliana-DapL

C.reinhardti-DapL

V.spinosum-DapL

C. trachomatis-DapL

A. thaliana-DapL

C. reinhardtii-DapL
-----------------------------MALINENFLKLKAGYLFPEIARRVK 25 -MKRNPHFVSLTKNYLFADLQKRVA 24 -----------MAKRVNTCKCVATPQEKIEYKTKVSRNSNMSKLQAGYLFPEIARRRS 47 MQLNVRSTASGARSSTRSRRMTAVVQAVAQRAGTIDVQRNENFGKLRAGYLFPEIARRRK 60

$$
\text { * : : } \text { LoOp }^{*} \mathrm{~B}^{* *} .:::^{*}
$$

AFTEGNPEAAQRLIRCGIGDVTEALPEAVRYAMHEAVDELGNRSTFKGYGPEQGYDFLRN 85 QFRLENPQH--TVINLSIGDTTQPLNASVAEAFASS IARLSSPTTCRGYGPDFGLPALRQ 82 AHLLKYPDA--QVISLGIGDTTEPIPEVITSAMAKKAHELSTIEGYSGYGAEQGAKPLRA 105 AHQEKNPDA--KIISLGIGDTTEPLPKYIADAMAKAAAGLATREGYSGYGAEQGQGALRE 118

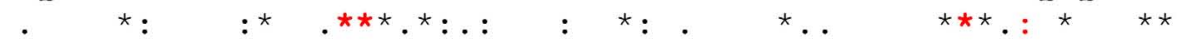

AIADNDYKARGLPIEADEIFISDGSKCDTGNILDIFGQGNTIAITDPVYPVYVDTNVMIG 145 KLSEDFYRGFV---DAKEIFISDGAKVDLFRLLSFFGPNQTVAIQDPSYPAYLDIARLTG 139 AIAKTFYGGLG--IGDDDVFVSDGAKCDISRLQVMFGSNVTIAVQDPSYPAYVDSSVIMG 163 AVASTFYGHAG--RAADEIFISDGSKCDIARIQMMFGSKPTVAVQDPSYPVYVDTSVMMG 176

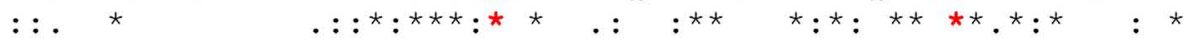

NTGEADEN-GAYAGLVYLKCTPENGFVPDIPQE-KADLIYLCYPNNPTGAVATRPQLEAW 203 AK----------EIIALPCLQENAFFPEFPEDTHIDILCLCSPNNPTGTVLNKDQLRAI 188 QTGQFNTDVQKYGNIEYMRCTPENGFFPDLSTVGRTDIIFFCSPNNPTGAAATREQLTQL 223 MTGDHNG--TGFDGIEYMVCNPDNHFFPDLSKAKRTDIIFFCSPNNPTGAAATRAQLTEL 234

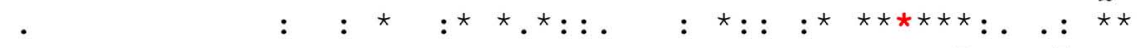$$
\text { Loop A }
$$

VKYARENGSVLLYDAAYEAFIQDPTIPHSIFEIEGARDCAIEFRSFSKNGGFTGVRCAYV 263 VHYAIEHEILILFDAAYSTFISDPSLPKSIFEIPDARFCAIEINSFSKPLGFAGIRLGWT 248 VEFAKKNGS I IVYDSAYAMYMSD-DNPRS IFEI PGAEEVAMETASFSKYAGFTGVRLGWT 282 VNFARKNGS ILVYDAAYALYISNPDCPKTIYEIPGADEVAIETCSFSKYAGFTGVRLGWT 294

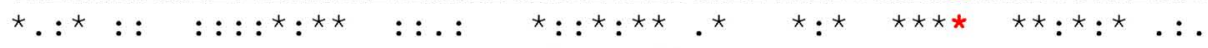
Loop $\mathrm{C}^{*}$

VIPKSLMGRKKNGEAQALHPLWSRRHSTKFNGASYIVQKGAEALYTDEGKSQTKALIEHY 323 VIPQELT----YADGHFVIQDWERFLSTTFNGASIPAQEAGVAGLS---ILPQLEAIHYY 301 VIPKKLL----YSDGFPVAKDFNRI ICTCFNGASNISQAGALACLTPEGLEAMHKVIGFY 338 VVPKALK----YANGEPVHADWNRVMTTCFNGASNIVQAGGLACLQPEGLKEMNAMIKFY 350

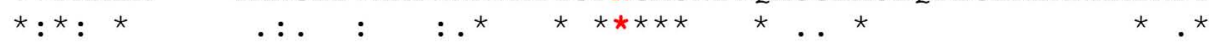

MGNAALLVEACKNAGLSVFGGVNAPYVWVGCPAG-LTSWQMFDKMLNEANVVITPGSGFG 382 RENSDLLRKALLATGFEVFGGEHAPYLWVKPTQANISDRDLFDFFLREYHIAITPGIGFG 361 KENTNIIIDTETSLGYDVYGGKNAPYVWVHFPN--QSSWDVFAEILEKTHVVTTPGSGFG 396 KENAQILKTTETEMGFSVYGGDDAPYIWVGFPG--KPSWDVEAEILERCNIVTTPGSGYG 408

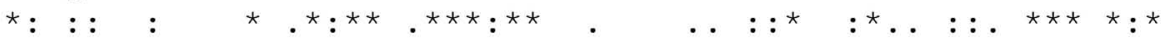

SAGEGYFRISAFNSRANVEEVCRRIAALK---- 411 RSGSGFVRFSSLGKREDILAACERLQMAPALQS 394 PGGEGFVRVSAFGHRENILEACRRFKQ------ 423

PAGEGFVRASAFGSRENILEAVRRFKEAYGKRN 441

$$
{ }^{\star} .{ }^{\star}:{ }^{\star}{ }^{\star}::{ }^{\star}::: .{ }^{\star}:
$$

FIGURE 8 | Protein sequence alignment of DapL from V. spinosum, $C$. trachomatis, $A$. thaliana, and $C$. reinhardtii generated using the ClustalW server (http://www.ebi.ac.uk/Tools/msa/clustalw2/). Putative active-site residues, based on the structural studies of the ligand bound AtDapL and CrDapL enzymes, are shown in red. The loop regions correspond to the key loops that line the active site, where the asterisk refers to the loop contributing to the active site of the opposing monomer. The ClustalW scores relative to the $V$. spinosum sequence were: $v s$. C. reinhardtii $=49$ (length $=443$ residues, identity); vs. $A$. thaliana $=45$ (length $=426$ residues); vs. $C$. trachomatis $=39$ (length $=394$ residues). thermoautotrophicus (NP_275195), Geobacter sulfurreducens (NP_951224), Leptospira interrogans (YP_002757), Nostoc PCC 7120 (NP_488367), Gloeobacter violaceus PCC 7421
(NP_927054), Methanosaeta thermophila PT (YP_843230), Methanospirillum hungatei JF1 (YP_504354), Dehalococcoides CBDB1 (YP_307791), Methanococcoides burtonii DSM 6242 

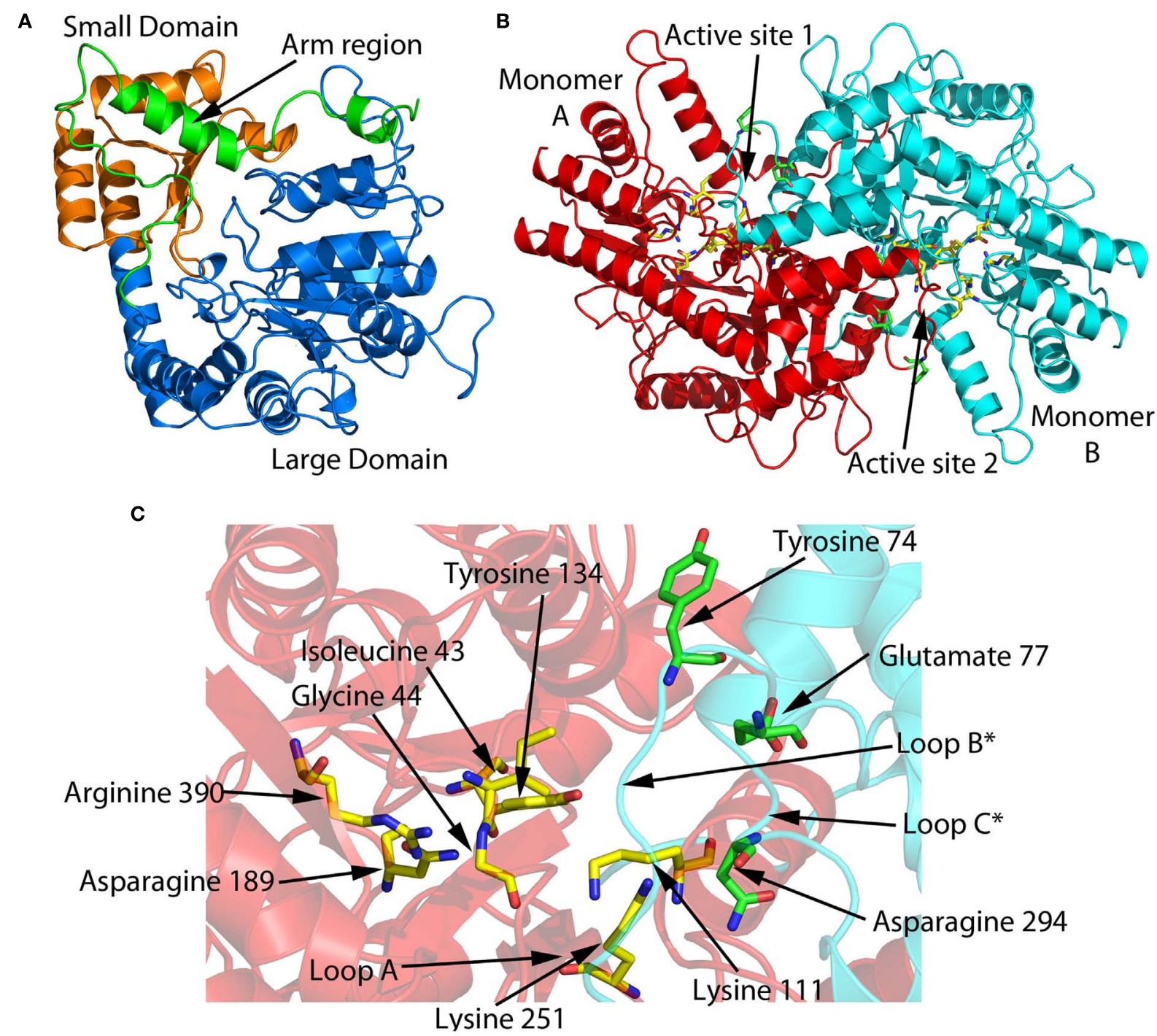

FIGURE $9 \mid$ VsDapL homology model generated with the Swiss-Model Protein Modeling Server (Arnold et al., 2006) using the CrDapL apo structure as a template (PDB id: 30GU Dobson et al., 2011; Hudson et al., 2011a, BLAST identity $=\mathbf{5 1 \%}$ (207/404 residues), OMEAN4 score $=\mathbf{0 . 6 7 3}$ ). (A) The domain structure of the monomer. Note that, as seen in Figure 8, VsDapL has a truncated
$\mathrm{N}$-terminal arm region compared to $\mathrm{CrDapL}$ and AtDapL. (B) Assuming a dimeric structure, which is required to place the conserved active site resides (highlighted in red bold Figure 8), the two active sites of the dimer are shown. (C) A close up view of active site 1 showing the conserved active site residues and the loops lining the active site (as per Figure 8).
(YP_565702), Methanosarcina barkeri Fusaro (YP_306095), Methanosarcina acetivorans C2A (NP_616639), Syntrophobacter fumaroxidans MPOB (YP_844192), Planctomyces brasiliensis DSM 5305 (YP_004269630), Planctomyces limnophilus DSM 3776 (YP_003632005), Verrucomicrobiae bacterium DG1235 (ZP_05058547). The accession number for the Yersinia pseudotuberculosis IP 32953 aspartate aminotransferase (AspC) is (CAH20674).

A homology model of the $V s$ DapL protein was generated using the Swiss-Model Protein Modeling Server (Arnold et al., 2006) using the CrDapL apo structure as a template (PDB id: 3QGU; Dobson et al., 2011). The model was examined by hand for clashes and appropriate geometry using the visualization software COOT (Emsley and Cowtan, 2004).

\section{PCR AMPLIFICATION AND CLONING OF THE V. SPINOSUM dapL AND mUrE OPEN READING FRAMES}

The full length ORFs annotated by the locus tags VspiD_0101000 12510 (dapL, L,L-diaminopimelate aminotransferase) and VspiD_010100019130 (murE, UDP-N-acetylmuramoylalanyl-Dglutamyl-2,6-meso-diaminopimelate ligase) were amplified by PCR. The ORFs were amplified using: $12 \mathrm{pmol}$ of forward and reverse primers, $1 \mathrm{mM} \mathrm{MgSO}_{4}, 0.5 \mathrm{mM}$ of each of the four deoxynucleotide triphosphates, 0.5 ng of genomic DNA and 1 unit 
Table 2 | Activity of $V s$ DapL with various amino donors and amino acceptors.

\begin{tabular}{llll}
\hline Amino donor & $\begin{array}{l}\text { Relative } \\
\text { activity (\%) }\end{array}$ & Amino acceptor & $\begin{array}{l}\text { Relative } \\
\text { activity (\%) }\end{array}$ \\
\hline L,L-diaminopimelate & 100 & 2-Ketoglutarate & 100 \\
meso-Diaminopimelate & $1.6 \pm 0.35$ & Pyruvate & $8.1 \pm 0.3$ \\
L-Lysine & $3.0 \pm 0.2$ & Oxaloacetate & $7.4 \pm 0.15$ \\
L-Ornithine & $5.1 \pm 0.2$ & Oxovalerate & $7.9 \pm 0.15$ \\
\hline
\end{tabular}

The assay measures the production of dihydroquinazolium at $440 \mathrm{~nm}$ using the ortho-aminobenzaldehyde assay. The assays pertaining to each amino donor/acceptor combination was done in triplicates, the relative activity is based on the activity of the enzyme with L,L-diaminopimelate and 2-ketoglutarate as substrates.

of Platinum Pfx DNA polymerase (Invitrogen Corporation, Carlsbad, CA, USA) using the following PCR conditions: 1 cycle at $94^{\circ} \mathrm{C}$ for $2 \mathrm{~min}$, followed by 30 cycles of $94^{\circ} \mathrm{C}$ for $15 \mathrm{~s}, 60^{\circ} \mathrm{C}$ for $30 \mathrm{~s}$ and $72^{\circ} \mathrm{C}$ for $2 \mathrm{~min}$. The forward and reverse primers used to amplify the ORFs were $V s d a p L \mathrm{~F}$ and $V s d a p L \mathrm{R}$ for the dapL ORF and VsmurE $\mathrm{F}$ and VsmurE R for the murE ORF. The nucleotide sequences of the primers are listed in Table 4 . The underlined sequence represents the restriction enzyme sites used to facilitate cloning of the ORF while the bolded and italicized sequences represent initiation and termination codons. For cloning, the dapL PCR fragment was digested with EcoRI and SalI and ligated into the plasmid pET30a (EMD Biosciences, Gibbstown, NJ, USA) to produce the plasmid pET30a $+V s d a p L$. The murE PCR fragment was ligated into the plasmid pET100D/topo (Invitrogen Corporation, Carlsbad, CA, USA) to produce the plasmid pE100D + VsmurE. The recombinant protein derived from this plasmid carries a hexahistidine tag derived from pET30a and pET100D plasmids at the amino terminus. To confirm the fidelity of the PCR reactions, the dapL ORF was sequenced from pET30a using the T7 promoter and the T7 terminator primer (Table 4). The murE ORF was sequenced from $\mathrm{pET} 100 \mathrm{D}$ using the T7 promoter and T7 R primer (Table 4). Both the dapL and murE ORFs are $100 \%$ identical to the sequences deposited in the Integrated Microbial Genomes (IMG) public database ${ }^{3}$.

\section{FUNCTIONAL COMPLEMENTATION PLASMID CONSTRUCTS}

The plasmids used for functional complementation of the $E$. coli $\Delta$ dapD/dapE double mutant and the E. coli murE mutant were produced by sub-cloning the XbaI and SalI fragment from $\mathrm{pET} 30 \mathrm{a}+V$ sdapL and the pET100D + VsmurE plasmids into pBAD33 to produce pBAD33 + VsdapL and pBAD33 + VsmurE (Guzman et al., 1995). The fusion proteins produced from the pBAD33 constructs are identical to the proteins produced from the pET30a and pET100D constructs.

\section{FUNCTIONAL COMPLEMENTATION OF THE E. COLI dapD/E AND murE MUTANTS}

The E. coli mutant AOH1 ( $\Delta$ dapD::Kan2, dapE6) was transformed with pBAD33 or plasmids harboring dapL orthologs

${ }^{3}$ http://img.jgi.doe.gov/cgi-bin/w/main.cgi
Table 3 | Plasmids and strains used in this study.

\begin{tabular}{ll}
\hline Plasmid/strains & Vendor/Reference \\
\hline pET30A & Novagen, USA \\
pET100D/Topo & Invitrogen, USA \\
pBAD33 & Guzman et al. (1995) \\
pBAD33 + AtdapL & Hudson et al. (2006) \\
pBAD33 + CrdapL & Dobson et al. (2011) \\
pET30A + VsdapL & This study \\
pET100D + VsmurE & This study \\
pBAD33 + VsdapL & This study \\
pBAD33 + VsmurE & This study \\
Verrucomicrobium spinosum DSM 4136 & Schlesner (1987) \\
5-alpha competent cells & New England Biolabs, USA \\
BL21 codon plus RIPL & Agilent Technologies, USA \\
AOH1 ( $\Delta$ dapD/dapE) & Hudson et al. (2006) \\
TKL-11 (murE) & Lugtenberg and van \\
& Schijndel-van Dam (1972) \\
\hline
\end{tabular}

Table 4 | List of Primers used for PCR amplification, cloning and nucleotide sequencing.

Primer name Sequence (from $5^{\prime}$ to $3^{\prime}$ )

Vsdapl F CCCCGAATTCATGGCCCTCATCAACGAAAACTTCCTCAAG

Vsdapl R CCCCGTCGACCTACTTCAGCGCGGCGATACGGCGGCAGAC

VsmurE F CACCATGACCATTTTGCGCGATCTTATCGAGGGT

VsmurE R GTCGACTCACTGACGGTCATCCCTCCTTTGGCGTGC

T7 promoter TAATACGACTCACTATAGGG

T7 R TAGTTATTGCTCAGCGGTGG

T7 terminator TATGCTAGTTATTGCTCAG

The underlined sequences represent restriction enzyme sites. The bolded and italicized sequences are denoted as the start and stop codons for the open reading frames (ORFs).

from $V$. spinosum (pBAD33 + VsdapL), Arabidopsis thaliana $(\mathrm{pBAD} 33+$ AtdapL), and $C$. reinhardtii (pBAD33 + CrdapL). Transformants were selected on LB agar medium supplemented with $50 \mu \mathrm{g} \mathrm{mL}^{-1}$ DAP and $34 \mu \mathrm{g} \mathrm{mL}^{-1}$ chloramphenicol. Ten individual colonies from the vector only and the recombinant plasmid were then replica-plated onto LB medium plus $0.2 \%$ (w/v) arabinose with or without $50 \mu \mathrm{g} \mathrm{mL}^{-1}$ DAP. The cultures were grown at $30^{\circ} \mathrm{C}$ for $24 \mathrm{~h}$. The E. coli murE mutant (TKL-11) (thr-1, leuB6 (Am), murE1, fhuA21, codA1, lacY1, tsx-95, glnV44 (AS), $\lambda^{-}$, pyrF101, his-108, thyA6, argG66, ilvA634, thi-1, deoC1) was transformed with pBAD33 or pBAD33 + VsmurE (Table 3). Transformants were selected on LB agar medium supplemented with $50 \mu \mathrm{g} \mathrm{mL}^{-1}$ thymine and $34 \mu \mathrm{g} \mathrm{mL}^{-1}$ chloramphenicol at $30^{\circ} \mathrm{C}$. Ten individual colonies from the vector only and recombinant plasmid transformation were replica-plated onto LB medium plus $0.2 \%(\mathrm{w} / \mathrm{v})$ arabinose and $50 \mu \mathrm{g} \mathrm{mL}^{-1}$ thymine. The cultures were grown at 30 and $42^{\circ} \mathrm{C}$ for $24 \mathrm{~h}$ to assess functional complementation.

\section{PROTEIN EXPRESSION AND PURIFICATION OF VsDapL}

The E. coli BL21-CodonPlus-RIPL strain was transformed with the plasmid pET30a $+V s d a p L$ and grown in LB broth containing 
$50 \mu \mathrm{g} \mathrm{mL}^{-1}$ kanamycin and $34 \mu \mathrm{g} \mathrm{mL}^{-1}$ chloramphenicol at $37^{\circ} \mathrm{C}$ to an $\mathrm{OD}_{600}$ of 0.5 . Protein expression was induced in $1.0 \mathrm{~L}$ of culture using isopropyl $\beta$-D-1-thiogalactopyranoside to a final concentration of $0.5 \mathrm{mM}$ for $6 \mathrm{~h}$ at $20^{\circ} \mathrm{C}$. The cell pellet was lysed by sonication in a solution of $50 \mathrm{mM}$ sodium phosphate $(\mathrm{pH} 8.0)$ and $300 \mathrm{mM} \mathrm{NaCl}$. The soluble extract was incubated with $1 \mathrm{~mL}$ bed volume of Talon Metal Affinity Resin (Clontech, Mountain View, CA, USA) for $30 \mathrm{~min}$ at $4^{\circ} \mathrm{C}$. The resin was washed five times with $30 \mathrm{~mL}$ of sonication buffer containing $10 \mathrm{mM}$ imidazole $(\mathrm{pH} 8.0)$ for $15 \mathrm{~min}$ each. The enzyme was eluted with $10 \mathrm{~mL}$ of sonication buffer containing $250 \mathrm{mM}$ imidazole $(\mathrm{pH}$ 8.0). The pure protein was concentrated in an Amicon Ultra 10,000 molecular weight cutoff filter unit replacing the elution buffer with $100 \mathrm{mM}$ HEPES-KOH containing $1 \mathrm{mM}$ DTT, $2 \mathrm{mM}$ EDTA ( $\mathrm{pH}$ 7.6). The recombinant protein was stored in 50\% glycerol. Protein concentration was measured using the Bradford assay with bovine serum albumin as the standard (Bradford, 1976).

\section{L,L-DIAMINOPIMELATE AMINOTRANSFERASE ENZYME ORTHO-AMINOBENZALDEHYDE ASSAY}

The ortho-aminobenzaldehyde assay measured the formation of THDPA from $L, L$-DAP using ortho-aminobenzaldehyde, which forms a dihydroquinazolium adduct with a maximum absorbance

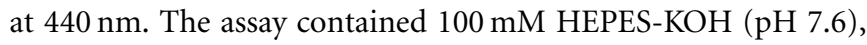
$0.5 \mathrm{mM}$ amino donor, $2 \mathrm{mM}$ amino acceptor and $1.25 \mathrm{mM}$ orthoaminobenzaldehyde and $10.0 \mu \mathrm{g}$ of purified recombinant $V s \mathrm{DapL}$ enzyme in a $0.5 \mathrm{~mL}$ reaction. The reactions were incubated at $30^{\circ} \mathrm{C}$ and the change in absorbance was measured continuously at $440 \mathrm{~nm}$ using a Beckman DU640 spectrophotometer.

\section{REFERENCES}

Arnold, K., Bordoli, L., Kopp, J., and Schwede, T. (2006). The SWISSMODEL workspace: a web-based environment for protein structure homology modeling. Bioinformatics 22, 195-201.

Baizman, E. R., Branstrom, A. A., Longley, C. B., Allanson, N., Sofia, M. J., Gange, D., and Goldman, R. C. (2000). Antibacterial activity of synthetic analogues based on the disaccharide structure of moenomycin, an inhibitor of bacterial transglycosylase. Microbiology 146, 3129-3140.

Bradford, M. M. (1976). A rapid and sensitive method for the quantification of microgram quantities of protein utilizing the principle of protein dye binding. Anal. Biochem. 72, 248-254.

Cox, R. J. (1996). The DAP pathway to lysine as a target for antimicrobial agents. Nat. Prod. Rep. 13, 29-43.

Dobson, R. C. J., Girón, I., and Hudson, A. O. (2011). L,Ldiaminopimelate aminotransferase from Chlamydomonas reinhardtii: a target for algaecide

\section{MESO-DIAMINOPIMELATE DEHYDROGENASE ACTIVITY}

Ddh activity was assessed using oxidative deamination. The assay consisted of $0.5 \mathrm{mM} m$-DAP, $0.5 \mathrm{mM} \mathrm{NADP}^{+}$, and $1 \mathrm{mg}$ of crude soluble $V$. spinosum extract or $10 \mu \mathrm{g}$ of pure recombinant mesodiaminopimelate dehydrogenase from $C$. thermocellum (Hudson et al., 2011b) in $100 \mathrm{mM}$ glycine- $\mathrm{KOH}$ ( $\mathrm{pH} \mathrm{10.5)} \mathrm{or} 100 \mathrm{mM}$ HEPES-KOH ( $\mathrm{pH} 7.6$ ) in a final volume of $0.5 \mathrm{~mL}$. The production of NADPH was measured continuously at $340 \mathrm{~nm}$ at $30^{\circ} \mathrm{C}$ using a Beckman DU640 spectrophotometer

\section{ACKNOWLEDGMENTS}

This research was supported by a United States National Science Foundation (NSF) award to AOH (MCB-1120541). Victoria R. Nachar was supported by an Undergraduate Summer Research Fellowship by the College of Science at RIT. Victoria R. Nachar and Francisco C. Savka are enrolled in the Biology Program and SEM is enrolled in Bioinformatics Program at RIT. Victoria R. Nachar, Francisco C. Savka, and Sean E. McGroty was supported by the NSF award (MCB-1120541) to André O. Hudson. Renwick C. J. Dobson acknowledges the CR Roper Bequest for Fellowship support, the New Zealand Royal Society Marsden Fund (UOC1013) for funding support and that this material is based upon work supported in part by the U. S. Army Research Laboratory and the U.S. Army Research Office under contract/grant number W911NF-111-0481. Katherine A. Donovan and Rachel A. North acknowledge the University of Canterbury Summer Scholarship Scheme. André O. Hudson thanks Naomi L. Ward form the University of Wyoming for graciously providing the V. spinosum strain/genomic DNA. The authors thank Michael A. Savka (RIT) for helpful discussions and revisions of the manuscript.

novel diaminopimelate biosynthesis pathway in prokaryotes identifies a diverged form of L,Ldiaminopimelate aminotransferase. J. Bacteriol. 190, 3256-3263.

Hudson, A. O., Girón, I., and Dobson, R. C. J. (2011a). Crystallization and preliminary X-ray diffraction analysis of L,L-diaminopimelate aminotransferase (DapL) from Chalmydomonas reinhardtii. Acta Crystallogr. Sect. F Struct. Biol. Cryst. Commun. 67, 140-143.

Hudson, A. O., Klartag, A., Gilvarg, C., Dobson, R. C. J., Marques, F. G., and Leustek, T. (2011b). Dual diaminopimelate biosynthesis pathways in Bacteroides fragilis and Clostridium thermocellum. Bochim. Biophys. Acta 1814, 1162-1168.

Hudson, A. O., Singh, B. K., Leustek, T., and Gilvarg, C. (2006). An L,Ldiaminopimelate aminotransferase defines a novel variant of the lysine biosynthesis pathway in plants. Plant Physiol. 140, 292-301.

Hutton, C. A., Perugini, M. A., and Gerrard, J. A. (2007). Inhibition of lysine biosynthesis: an evolving antibiotic strategy. Mol. Biosyst. 3, 458-465.
Jones, D. T., Taylor, W. R., and Thornton, J. M. (1992). The rapidgeneration of mutationdatamatrices from proteinsequences. Comput. Appl. Biosci. 8, 275-282.

Liu, Y., White, R. H., and Whitman, W. B. (2010). Methanococci use the diaminopimelate aminotransferase (DapL) pathway for lysine biosynthesis. J. Bacteriol. 192, 3304-3310.

Lugtenberg, E. J. J., and van Schijndelvan Dam, A. (1972). Temperaturesensitive mutants of Escherichia coli K-12 with low activity of the diaminopimelic acid adding enzyme. J. Bacteriol. 110, 41-46.

McCoy, A. J, Adams, N. E., Hudson, A. O., Gilvarg, C., Leustek, T., and Maurelli, A. T. (2006). L,LDiaminopimelate aminotransferase a trans-kingdom enzyme shared by Chlamydia and plants for synthesis of diaminopimelate/lysine. Proc. Natl. Acad. Sci. U.S.A. 103 , 17909-17914.

Mills, W. R., and Wilson, K. G. (1978). Amino acid biosynthesis in isolated pea chloroplast: metabolism of labeled aspartate and sulfate. FEBS Lett. 92, $129-132$. 
Nishida, H. M., Nishiyama, N., Kobayashi, T., Kosuge, T., Hoshimo, T., and Yamane, H. (1999). A prokaryotic gene cluster involved in synthesis of lysine through the amino adipate pathway: a key to the evolution of amino acid biosynthesis. Genome Res. 9, 1175-1183.

Rambaut, A. (2009). FigTree: Tree Figure Drawing Tool. Version 1.3.1, Institute of Evolutionary Biology, University of Edinburgh. Available at: http://tree.bio.ed.ac.uk/

Sait, M., Kamneva, O. K., Fay, D. S., Kirienko, N. V., Polek, J., ShirasuHiza, M. M., and Ward, N. L. (2011). Genomic and experimental evidence suggests that Verrucomicrobium spinosum interacts with eukaryotes. Front. Microbiol. 2:211. doi:10.33389/fmicb.2011.00211

Schlesner, H. (1987). Verrucomicrobium spinosum gen. nov., sp. nov.: a fimbriated prosthecate bacterium. Syst. Appl. Microbiol. 10, 54-56.

Schlesner, H., Jenkins, C., and Staley, J. T. (2006). The phylum Verrucomicrobia: a phylogenetically heterogeneous bacterial group. Prokaryotes 7 , 881-896.

Schrumpf, B., Schwarzer, A., Kalinowski, J., Puhler, A., Eggeling, L., and
Sahm, H. (1991). A functionally split pathway for lysine synthesis in Corynebacterium glutamicum. J. Bacteriol. 173, 4510-4516.

Tamura, K., Peterson, D., Peterson, N., Stecher, G., Nei, M., and Kumar, S. (2011). MEGA5: molecular evolutionary genetics analysis using maximum likelihood, evolutionary distance, and maximumparsimony. Mol. Biol. Evol. 28, 2731-2739.

Velasco, A. M., Leguina, J. I., and Lazcano, A. (2002). Molecular evolution of the lysine biosynthetic pathways. J. Mol. Evol. 55, 445-459.

Wagner, M., and Horn, M. (2006). The Planctomycetes, Verrucomicrobia, Chlamydiae and sister phyla comprise a superphylum with biotechnological and medical relevance. Curr. Opin. Biotechnol. 17, 241-249.

Watanabe, N., Clay, M. D., van Belkum, M. J., Cherney, M. M., Vederas, J. C., and James, M. N. (2008). Mechanism of substrate recognition and PLP-induced conformational changes in L,L-diaminopimelate amionotransferase from Arabidopsis thaliana. J. Mol. Biol. 384, 1314-1329.

Watanabe, N., Clay, M. D., van Belkum, M. J., Fan, C., Vederas, J. C., and
James, M. N. (2011). The structure of L,L-diaminopimelate aminotransferase from Chlamydia trachomatis: implications for its broad substrate specificity. J. Mol Biol. 19, 649-660.

Watanabe, N., Clay, M. D., van Belkum, M. J., Marcus, S. L., Flegel, M. D., Clay, M. D., Deyholos, M. K., Vederas, J. C., and James, M. N. (2007). Crystal structure of L,L-diaminopimelate aminotransferase from Arabidopsis thaliana: a recently discovered enzyme in the biosynthesis of L-Lysine by plants and Chlamydia. J. Mol. Biol. 371, 685-702.

Whelan, S., and Goldman, N. (2001). A general empirical model of protein evolution derived from multiple protein families using a maximumlikelihood approach. Mol. Biol. Evol. $18,691-699$.

Yoneyama, H., Hori, H., Lim, S., Murata, T., Ando, T., Isogai, E., and Katsumata, R. (2011). Isolation of mutant auxotrophic for L-Alanine and identification of three major aminotransferases that synthesize LAlanine in Escherichia coli. Biosci. Biotechnol. Biochem. 75, 930-938.

Conflict of Interest Statement: The authors declare that the research was conducted in the absence of any commercial or financial relationships that could be construed as a potential conflict of interest.

Received: 14 January 2012; accepted: 02 May 2012; published online: 25 May 2012.

Citation: Nachar VR, Savka FC, McGroty SE, Donovan KA, North RA, Dobson RCJ, Buckley LJ and Hudson $A O$ (2012) Genomic and biochemical analysis of the diaminopimelate and lysine biosynthesis pathway in Verrucomicrobium spinosum: identification and partial characterization of $L, L$-diaminopimelate aminotransferase and UDP-N-acetylmuramoylalanylD-glutamyl-2,6-meso-

diaminopimelate ligase. Front. Microbio. 3:183. doi: 10.3389/fmicb.2012.00183

This article was submitted to Frontiers in Evolutionary and Genomic Microbiology, a specialty of Frontiers in Microbiology.

Copyright () 2012 Nachar, Savka, McGroty, Donovan, North, Dobson, Buckley and Hudson. This is an openaccess article distributed under the terms of the Creative Commons Attribution Non Commercial License, which permits non-commercial use, distribution, and reproduction in other forums, provided the original authors and source are credited. 